\title{
Blood clotting and fibrinolysis in pregnancy
}

\author{
P. W. HowIE \\ M.D., M.R.C.O.G. \\ MRC Unit of Reproductive Biology, Edinburgh
}

\begin{abstract}
Summary
During normal pregnancy, the concentrations of many of the clotting factors rise, thereby increasing the potential to generate fibrin. There is aso evidence of increased thrombin activity during normal pregnancy which sharply increases during placental separation. Antithrombin III, the main inhibitor of thrombin and activated factor $X$, shows no compensatory rise during pregnancy but increases during the puerperium.

Plasminogen and antiplasmin concentrations rise during pregnancy but systemic fibrinolytic activity, as measured by the euglobulin lysis time, is markedly depressed during pregnancy; the reduced fibrinolytic activity returns to non-pregnant values very soon after delivery. The loss of fibrinolytic activity is presumed to be loss of plasminogen activator, because when this is added in excess in the urokinase sensitivity test, the fibrinolytic response is normal. The capacity for localized fibrinolytic activity is not lost, however, because fibrinolytic degradation products are slightly raised during pregnancy.

The overall pattern is one of increased coagulant and reduced fibrinolytic capacity during pregnancy which may protect the pregnant woman against the haemostatic challenge of placental separation.
\end{abstract}

\section{Introduction}

The coagulation and fibrinolytic enzyme systems play an essential role in maintaining the integrity of the vascular tree. When a blood vessel is damaged, the coagulation system arrests the haemorrhage and the vessel blockage is subsequently cleared by fibrinolysis. Placental separation represents a profound haemostatic challenge so that it is not surprising that evolution has modified the coagulation and fibrinolytic systems during pregnancy to prevent undue bleeding. A description of how these systems are modified during the course of normal pregnancy now follows.

\section{Coagulation system in normal pregnancy}

The coagulation system (Fig. 1) can be activated in two ways, but both have the same final common pathway through the activation of factor $X$. The first method of activation is the intrinsic system which acts by surface contact when there is endothelial cell injury. This activates factor XII, or Hageman factor, which in turn activates a series of enzymatic reactions involving factors XI, IX, VIII and $\mathrm{X}$. The extrinsic system acts more rapidly when thromboplastin enters the circulation as a result of tissue damage; this, together with factor VII, can activate factor $\mathrm{X}$. Activated factor $\mathrm{X}$ combines with factor $\mathrm{V}$ and phospholipid derived from platelets to form the prothrombin converting principle. This is responsible for prothrombin to thrombin conversion, which in turn acts on fibrinogen to form fibrin. It is well known that many of the clotting factors rise during pregnancy and these include fibrinogen,

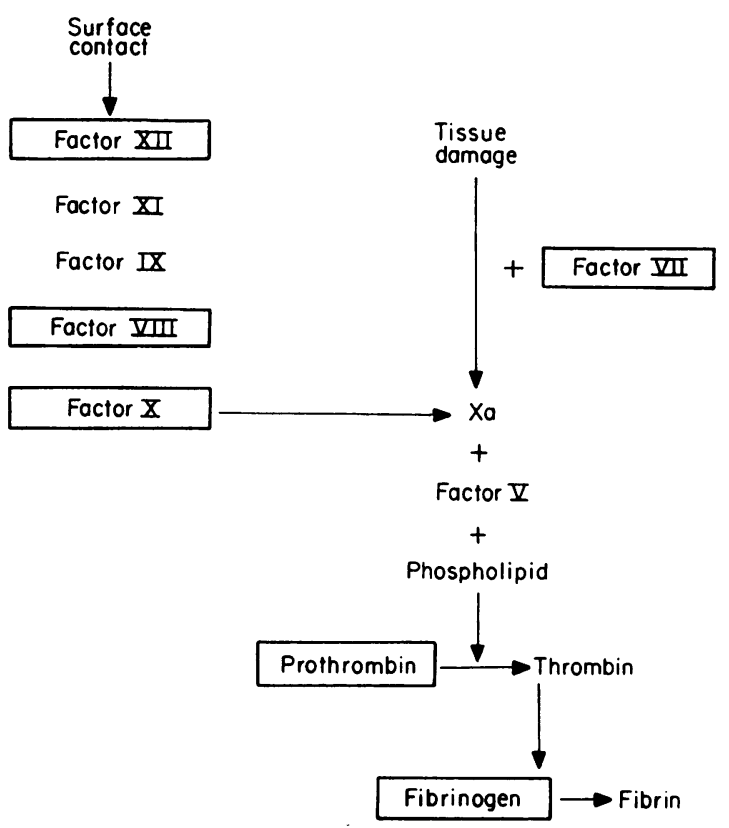

Fig. 1. Scheme of the coagulation mechanism showing which clotting factors (enclosed in boxes) are raised during normal pregnancy. 
prothrombin and factors VII, VIII, X and XII (Todd et al., 1965).

\section{Fibrinogen levels in pregnancy}

The coagulation factors tend to rise during the third trimester and the results of a recent study on the fibrinogen levels of 60 primigravidae is shown in Fig. 2 (Condie and Ogston, 1976). Before delivery the

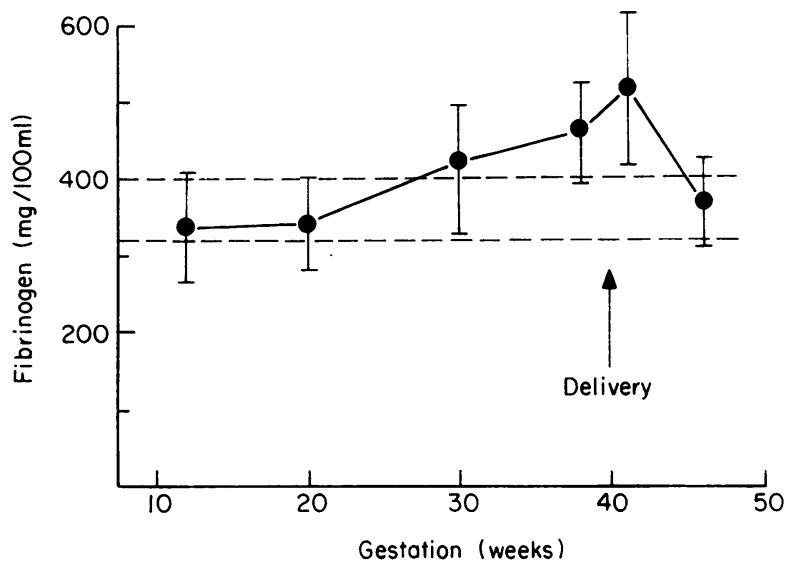

Fig. 2. Fibrinogen levels in pregnancy and the puerperium $(n=60)$. Mean levels \pm s.d. The dotted lines show the mean \pm 1 s.d. of a non-pregnant control group of women (from Condie and Ogston (1976) by permission of the Editor of the British Journal of Obstetrics and Gynaecology.

fibrinogen concentration is about $50 \%$ above the non-pregnant value. Bearing in mind the increase in circulating blood volume, there is about double the amount of fibrinogen available to the pregnant woman at delivery compared to the non-pregnant state. After delivery there is a secondary rise in fibrinogen followed by a return to normal.

\section{Thrombin activity in pregnancy}

The raised levels of clotting factors in pregnancy do not necessarily mean that there is increased clotting activity during pregnancy. There has been increasing interest in methods designed to measure thrombin activity more directly during pregnancy. One of the principal actions of thrombin is to split fibrinopeptides from negatively charged fibrinogen to form fibrin monomer, which becomes positively charged. Fibrin monomer and native fibrinogen then combine to form fibrin-polymer chains which may remain in soluble form within the circulation. These polymer chains may later be deposited as insoluble fibrin and, under the influence of factor XIII, there is cross-linking which renders the reaction irreversible. The presence of circulating soluble fibrin polymer can be detected by the relatively new technique of fibrinogen chromatography. It has been shown that there is a small rise of fibrin polymer complexes during normal pregnancy which is strong evidence of low-grade thrombin activity (McKillop et al., 1976).

\section{Factor VIII antigen-activity ratio}

When thrombin is formed, the first molecules act upon factor VIII to generate more coagulant activity and a second more vigorous burst of thrombin production. When thrombin activates factor VIII, the factor VIII molecule loses its coagulant activity but retains its antigenic potential. Therefore, when the factor VIII antigen-activity ratio rises, this is suggestive evidence of increased thrombin activity. A rise of factor VIII antigen-activity ratio has now been described (Van Royen and Ten Cate, 1973; Thornton and Bonnar, 1977) confirming the picture of increased thrombin-like activity in some women during normal pregnancy (Fig. 3). At the time of

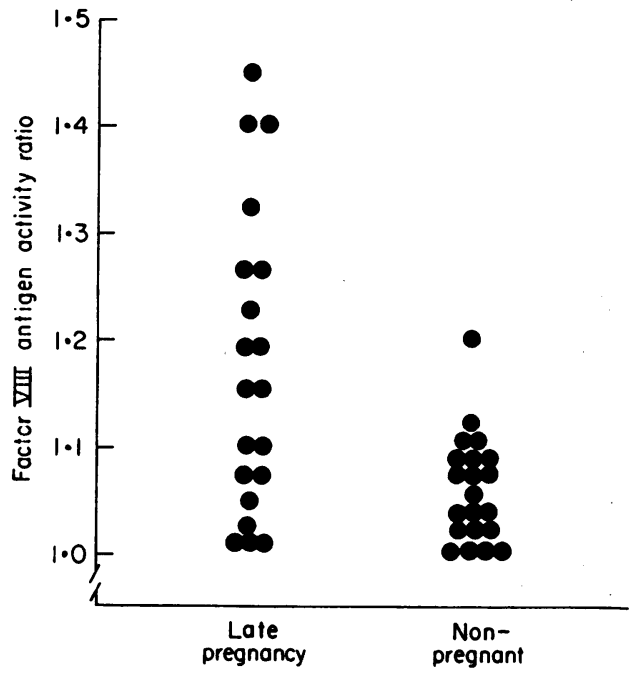

FIG. 3. Factor VIII antigen activity ratio in 20 pregnant and 20 non-pregnant women showing raised values in some of the pregnant women.

placental separation, studies on umbilical vein blood have shown a profound activation of coagulation (Bonnar, McNicol and Douglas, 1970).

\section{Antithrombin activity during pregnancy}

The main inhibitor of thrombin is antithrombin III which is an $\alpha_{2}$-globulin (mol. wt 60000 ) and which also inhibits activated factor $X$. The clinical importance of antithrombin III is demonstrated by the greatly increased risk of thrombo-embolism in 
patients with an inherited deficiency of this protein (Egeberg, 1965). Despite the increased potential to form thrombin in pregnancy, there is no compensatory rise in antithrombin III (Fig. 4) (Howie et al., 1975). Following delivery, antithrombin III levels rise during the first week of the puerperium which may reduce the risk of thromboembolism after delivery.

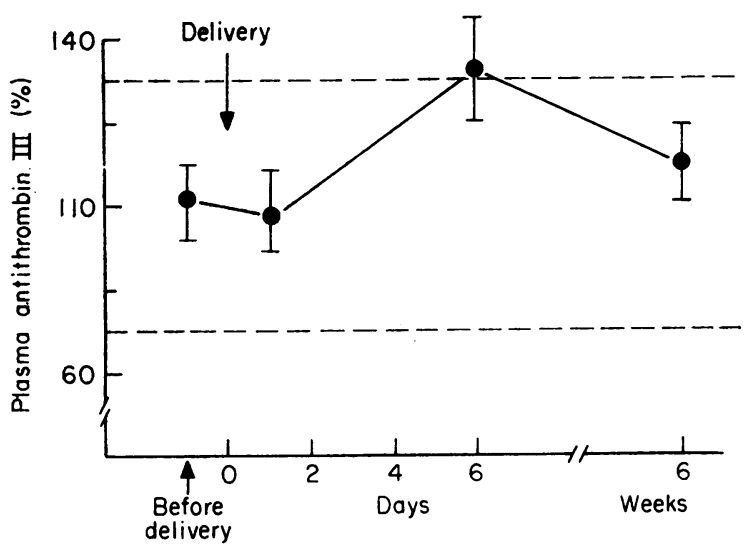

Fig. 4. Plasma antithrombin III before and after delivery $(n=15)$. Mean levels \pm s.d. The dotted lines show the non-pregnant range.

Antithrombin III is most probably identical to heparin co-factor whose activity is greatly potentiated by heparin. The antithrombin III values in pregnancy are consistent with the observation that there is relative resistance to heparin during pregnancy which rapidly subsides after delivery (Bonnar, 1976).

\section{Platelets}

The platelet count does not change during pregnancy but rises during the first week of puerperium before returning to non-pregnant values 6 weeks after delivery (Howie, Prentice and McNicol, 1971). Many tests of platelet function have been carried out during pregnancy, including platelet adhesiveness, platelet aggregation (Howie et al., 1971), serotonin release (Whigham et al., 1978) and platelet turnover (Wallenburg and van Kessel, 1978) but none of these tests deviated from the non-pregnant values. In summary, platelet behaviour shows little, if any, change during pregnancy from the nonpregnant state.

\section{Fibrinolytic system}

When fibrin is deposited, plasminogen is incorporated into the substance of the deposit to ensure its subsequent lysis. This lysis occurs when plasmin is formed following the action of activator upon the plasminogen. Activation may come through different routes, namely factor XII, tissue activator or vascular endothelium (Fig. 5). The inhibitors of activator

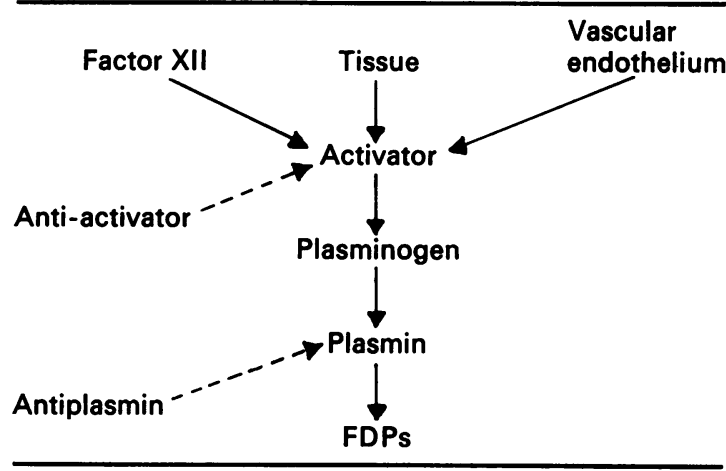

FIG. 5. Schematic representation of the fibrinolytic enzyme system.

and plasmin are present in high concentrations within the blood so that plasmin is rapidly neutralized in the free circulation. Within a fibrin clot, the situation is different; inhibitors are largely excluded so that activation of plasminogen to plasmin and lysis of fibrin to degradation products (FDPs) will take place when activator diffuses into the clot. Distinction must therefore be made between systemic and localized fibrinolytic activity.

\section{Euglobulin lysis time}

One of the most dramatic observations in pregnancy is the profound depression of fibrinolytic activity as measured by the euglobulin lysis time which returns to normal within $30 \mathrm{~min}$ of delivery (Shaper, MacIntosh and Kyobe, 1966). In this test (Fig. 6), the euglobulin precipitate is prepared which

1. Prepare euglobulin precipitate. Fibrinogen, plasminogen and activator (inhibitors excluded).

\section{Resuspend and clot with thrombin.}

3. Measure lysis time.

FIG. 6. The essential steps of the euglobulin lysis time.

contains fibrinogen, plasminogen and activator with the inhibitors excluded. The euglobulin precipitate is resuspended and clotted with thrombin and the lysis time is then measured. Since fibrinogen and plasminogen are present abundantly in pregnancy, the long lysis times in pregnancy are presumed to be due to diminished activator activity.

When activator activity is provided in excess, as in 
the urokinase sensitivity test (Fig. 7), the fibrinolytic response is normal (Howie et al, 1971). This is

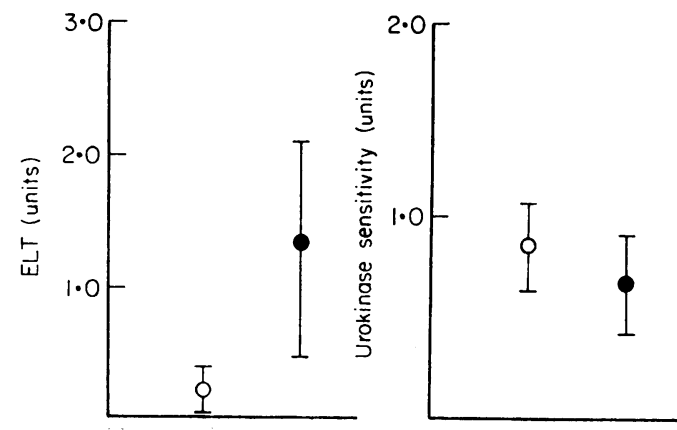

FIG. 7. The euglobulin lysis time (ELT) and the urokinase sensitivity test in late pregnancy $(n=12$; mean \pm s.d.) showing that the depressed fibrinolytic activity in pregnancy is corrected by the addition of activator (urokinase). controlling the coagulation and fibrinolytic systems in pregnancy. It has been shown that the return of fibrinolytic activity to normal depends more upon delivery of the placenta than delivery of the fetus (Astedt, 1972). Foley, Clayton and McNicol (1977) have recently compared the haemostatic mechanisms in umbilical vein and artery blood; the vein, in comparison to the artery, showed increased clotting and decreased fibrinolytic activity, suggesting a regulatory role for the placenta.

In summary, the pregnant woman shows an enhanced capacity to generate fibrin and an increase in thrombin-like activity without the balance of increased antithrombin activity. In addition, there is suppression of systemic fibrinolytic activity although the capacity for localized fibrinolysis is not abolished. This shift in balance may encourage more effective haemostasis but may also predispose the pregnant woman to the complications of thrombo-embolism or intravascular coagulation.
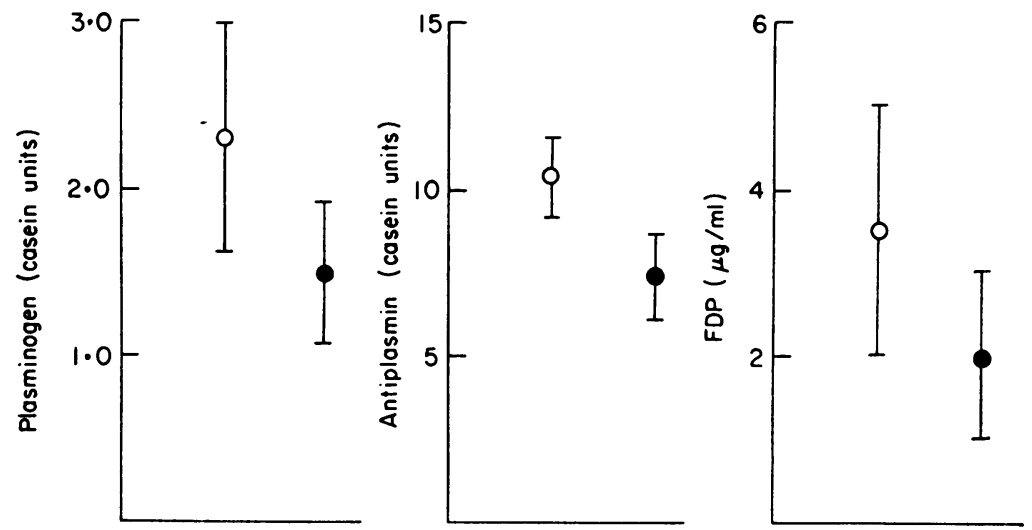

FIG. 8. Plasminogen, antiplasmin and FDP values $(n=12$; mean \pm s.d.) showing raised levels in late pregnancy $(O)$ as compared with non-pregnancy $(O)$.

further evidence that deficient activator activity is responsible for the decreased systemic fibrinolytic activity in pregnancy.

Plasminogen levels are raised in pregnancy (Bonnar, McNicol and Douglas, 1969) but these are counter-balanced by a similar rise in antiplasmin activity (Fig. 8). It is clear that localized fibrinolytic activity is not completely abolished because the capacity of the pregnant women to form FDPs is maintained and the levels in late pregnancy may be slightly raised over the non-pregnant range (Woodfield et al., 1968).

\section{Role of the placenta}

The placenta appears to play a critical role in

\section{References}

AstedT, B. (1972) Significance of the placenta in depression of fibrinolytic activity during pregnancy. Journal of Obstetrics and Gynaecology of the British Commonwealth, 79, 205.

BONNAR, J. (1976) Long-term self-administered heparin therapy for prevention and treatment of thrombembolic complications in pregnancy. In: Heparin: Chemistry and Clinical Usage (Ed. by Kakkar, V. \& Thomas, D.). Academic Press, London.

Bonnar, J., McNicol, G.P. \& Douglas, A.S. (1969) Fibrolytic enzyme system and pregnancy. British Medical Journal, 3, 387.

BonNar, J., McNicol, G.P. \& Douglas, A.S. (1970) Coagulation and fibrinolytic mechanisms during and after normal childbirth. British Medical Journal, 2, 200.

Condie, R.G. \& OGston, D. (1976) Sequential studies on components of the haemostatic mechanism in pregnancy 
with particular reference to the development of preeclampsia. British Journal of Obstetrics and Gynaecology, 83, 938.

EGEBERG, O. (1965) Inherited antithrombin deficiency causing thrombophilia. Thrombosis et diathesis haemorrhagica. 13, 516.

Foley, M.E., Clayton, J.K. \& McNicol, G.P. (1977) Haemostatic mechanisms in maternal, umbilical vein and umbilical artery blood at the time of delivery. British Journal of Obstetrics and Gynaecology, 84, 8.

Howie, P.W., Evans, K., Forbes, C.D. \& Prentice, C.R.M. (1975) The effects of stilboestrol and quinestrol upon coagulation and fibrinolysis during the puerperium. British Journal of Obstetrics and Gynaecology, 82, 968.

Howie, P.W., Prentice, C.R.M. \& McNicol, G.P. (1971) Coagulation, fibrinolysis and platelet function in preeclampsia, essential hypertension and placental insufficiency. Journal of Obstetrics and Gynaecology of the British Commonwealth, 78, 992.

McKillop, C., Howie, P.W., Forbes, C.D. \& Prentice, C.R.M. (1976) Soluble fibrinogen - fibrin complexes in pre-eclampsia. Lancet, i, 56.
Shaper, A.G., Macintosh, D.M. \& Kyobe, J. (1966) Fibrinolytic activity in pregnancy, during parturition and in the puerperium. Lancet, ii, 874 .

ThORNTON, C.A. \& BonNAR, J. (1977) Factor VIII-related antigen and factor VIII coagulant activity in normal and pre-eclamptic pregnancy. British Journal of Obstetrics and Gynaecology, 84, 919.

TodD, M.E., Thomson, J.H., Bowie, E.J.W. \& OWen, C.A. (1965) Changes in blood coagulation during pregnancy. Proceedings. Mayo Clinic, 40, 370.

VAN Royen, E.A. \& TEN CATE, J.W. (1973) Antigenbiological activity ratio for factor VIII in late pregnancy. Lancet, ii, 449.

Wallenburg, H.C.S. \& van Kessel, P.H. (1978) Platelet lifespan in normal pregnancy as determined by a nonradioisotopic technique. British Journal of Obstetrics and Gynaecology, 85, 33.

Whigham, K.A.E., Howie, P.W., Drummond, A.H. \& Prentice, C.R.M. (1978) Abnormal platelet function in pre-eclampsia. British Journal of Obstetrics and Gynaecology, 85, 28.

Woodfield, D.G., Cole, S.K., Allan, A.G.E. \& Cash, J.D. (1968) Serum fibrin degradation products throughout normal pregnancy. British Medical Journal, 4, 665. 